\title{
L-Edge Absorption Spectra of the Rare Earths: Assessment of the Single-Particle Picture
}

\author{
G. Materlik \\ School of Applied and Engineering Physics and Materials Science Center, Cornell University, Ithaca, New York \\ 14853, and Hamburger Synchrotronstrahlungslabor am Deutschen Elektronen-Synchrotron DESY, \\ D-2000 Hamburg 52, West Germany ${ }^{(a)}$ \\ and \\ J. E. Müller \\ Materials Science Center, Cornell University, Ithaca, New York 14853, and Institut für Festkörperforschung der \\ Kernforschungsanlage Jülich, D-5170 Jülich, West Germany (a) \\ and \\ J. W. Wilkins \\ Laboratory of Atomic and Solid State Physics and Materials Science Center, Cornell University, \\ Ithaca, New York 14853 \\ (Received 28 May 1982)
}

\begin{abstract}
X-ray $L$ absorption spectra were measured for the rare-earth metals Sm and from Gd to $\mathrm{Lu}$. The data are compared with single-particle band-structure calculations over an energy range of about $100 \mathrm{eV}$. The one-electron theory describes the white line and the near-edge structure strikingly well. At higher energies, although all features of the experimental spectra are reproduced by the calculation, the actual placement of the features shows discrepancies, which point to a limitation in the one-electron description.
\end{abstract}

PACS numbers: 78.70.Dm, 71.25.Pi, 71.45.Gm

The $L_{2,3} \mathrm{x}$-ray absorption spectra of the transition and rare-earth metals are distinguished by prominent peaks at threshold, the so-called white lines. These peaks, arising from transitions from atomic $2 p_{1 / 2}\left(L_{2}\right)$ and $2 p_{3 / 2}\left(L_{3}\right)$ core levels into a partially filled, large $d$ density of states are currently being studied in a number of ways. For example, electron-energy-loss studies on the $3 d$ transition metals have reported ${ }^{1}$ deviations from the two-to-one ratio of the $L_{3}$ to $L_{2}$ absorptions, expected on the basis of the initial-state degeneracies. Also $x$-ray absorption studies ${ }^{2-5}$ of the $L_{3}$ white line on rare-earth compounds exhibiting valence fluctuations have been used to deduce, simultaneously, relative valence and interatomic distances. ${ }^{3}$ Further, this pronounced edge feature provides a promising way partially to overcome the phase problem in structure determination of complex molecules by increasing the sensitivity of the method of anomalous dispersion. ${ }^{6}$

The interpretation of $\mathrm{x}$-ray absorption spectra (as well as most other types of core-electron spectra) is complicated by the creation of a core hole in one of the atoms of the solid. The magnitude of this effect for transition and rare-earth metals is not as yet known. Further, these spectra depend on the excited states of the electronic system, which are less well understood than the corresponding ground-state properties. ${ }^{7}$ As a basis for evaluating theories of spectroscopies, it is therefore essential to compare measured spectra with accurate single-particle calculations, which bear simple connection to the groundstate properties.

In this Letter we report both careful $x$-ray measurements of the $L$-edge absorption spectra of the heavy rare earths ( $\mathrm{Sm}$ and from $\mathrm{Gd}$ to $\mathrm{Lu}$ ) and detailed single-particle calculations which extend about $100 \mathrm{eV}$ above threshold. While the measured and calculated white lines exhibit a striking agreement, discrepancies are clearly noticeable in the higher-energy part of the spectra. There, the structure of the measured spectra is displaced to higher energy compared with that of the calculated spectra. This breakdown of the single-particle picture is most likely due to the fact that the excited electron sees a potential which is different from that appropriate for ground-state properties.

The spectra were recorded with three different types of nondispersive double-crystal spectrometers. All three systems used synchrotron radiation, one from the $12-\mathrm{GeV}$ Wilson Synchrotron at Cornell University, the other two from the storage ring DORIS in Hamburg. The signal-to-noise ratio was, when necessary, reduced by special combinations of symmetric and asymmetric sili- 
con crystals. ${ }^{8}$ Incident and transmitted intensities were recorded with ion chambers. In order to ensure that the height of each white line was not saturated by background radiation, single-photon counting with energy discrimination was also used for energies close to the absorption edge. The thickness of the samples (rolled foils of 99.9\% purity) was chosen to maximize the difference in transmitted intensity across the $L_{3}$ edge. The spectra for $\mathrm{Gd}, \mathrm{Tm}$, and $\mathrm{Yb}$ were also repeated with samples from different batches.

The calculations of the absorption spectra were based on a linearized version of the augmentedplane-wave method especially designed to cover large energy ranges. ${ }^{9}$ The input for the bandstructure calculation was a periodic, muffin-tin, ground-state potential, ${ }^{10}$ which is spherically symmetric inside nonoverlapping touching spheres centered on each atomic site and constant between the spheres. The resulting band energies $E_{\overrightarrow{\mathrm{k}}}$ and wave functions $\psi_{\vec{k}_{n}}$ were used in a single-particle calculation of the raw absorption spectrum

$$
\bar{\mu}_{c}(E) \propto \sum_{\overrightarrow{\mathrm{k}} n}|\langle c|r| \overrightarrow{\mathrm{k}} n\rangle|^{2} \delta\left(E+E_{c}-E_{\overrightarrow{\mathrm{k}}_{n}}\right),
$$

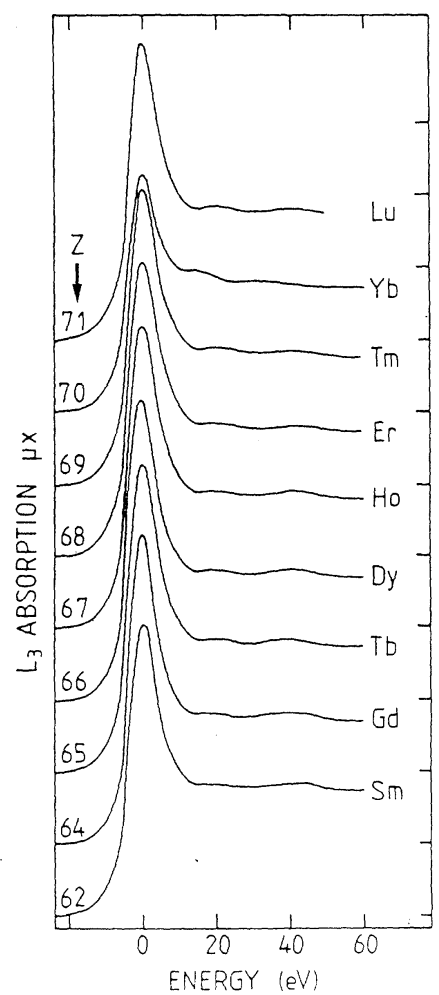

FIG. 1. Measured $L_{3}$ absorption spectra for rhombic hexagonal Sm, hep Gd, Tb, Dy, Ho, Er, Tm, and Lu, and $\mathrm{fcc} \mathrm{Yb}$. where the core level $c$ [in this case a $2 s\left(L_{1}\right)$ or a $2 p\left(L_{2,3}\right)$ level] has energy $E_{c}$. The raw spectrum was then broadened by first convoluting $\bar{\mu}_{c}(E)$ with a Lorentzian broadening function whose width was the sum of the inverse lifetimes of the core hole ${ }^{11}$ and of the excited electron, ${ }^{12}$ and then further convoluting with a Gaussian broadening function whose width was the instrumental resolution ( $1.5 \mathrm{eV}$ for $\mathrm{Gd})$.

Figure 1 presents the measured $L_{3}$ spectra for $\mathrm{Sm}$ and for $\mathrm{Gd}$ to $\mathrm{Lu}$. The vertical scales have been adjusted so that the high-energy absorption relative to the prethreshold background is the same for all the elements, thus permitting a visual determination of the relative magnitude of the white lines. For Sm and the hcp trivalent rare earths the ratio of the white-line peak to the high-energy absorption (both measured relative to the prethreshold background) is 2.3 within $5 \%$, while for fcc divalent $\mathrm{Yb}$ the ratio is 1.9 .

Figure 2 presents the measured and calculated spectra for the $L_{3}$ and $L_{1}$ edges of Gd. The weaker features above the white line have been represented in an expanded scale in order to facilitate the comparison between theory and experiment. The energy origins, each at the Fermi level, have been shifted to line up the thresholds which are determined by the inflection point of the onset slope. Observe that all the features of the

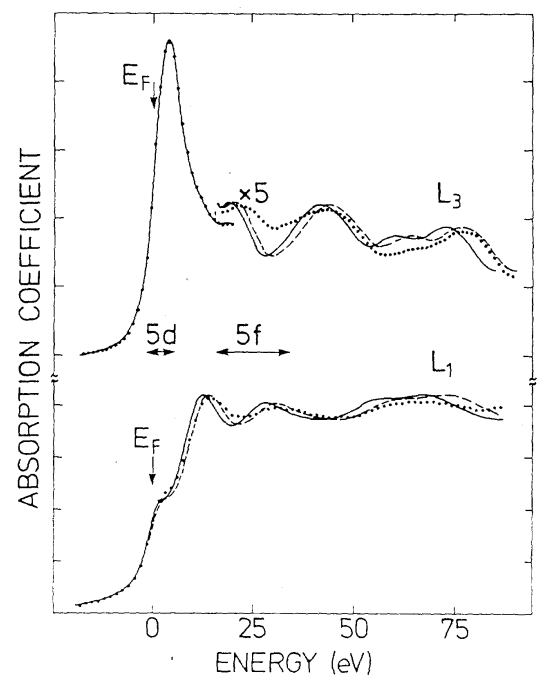

FIG. 2. $L_{3}$ and $L_{1}$ absorption edges for Gd. Comparison between the measured (dots) and calculated (solid lines) spectra. The dashed lines represent the calculated spectra with the energy axis rescaled by 1.05 for the $L_{3}$ spectra and by 1.07 for the $L_{1}$ spectra. The energy origins, each at the Fermi level, have been shifted to line up the thresholds. 
measured spectra are reproduced in the calculated spectra. However, the calculated features lie always at lower energies than the measured ones, i.e., it is not possible to align the highenergy features of the spectra. Agreement was not improved by using a self-consistent potential. Further, since the calculated energy levels increase with the number of basis functions (i.e., linear augmented plane waves), the number of basis functions was increased until the energies no longer changed (within $0.1 \mathrm{eV}$ ).

These discrepancies arise from the inadequacy of the ground-state potential to describe the highenergy states. ${ }^{13}$ An appropriate potential should include (i) the effect of the core hole in the excited atom, ${ }^{14}$ and (ii) the self-energy correction to the energy of the excited electron. ${ }^{15}$ The latter effect alone, we will argue next, cannot explain the observed discrepancy. One way to characterize this discrepancy is illustrated by the dashed lines in Fig. 2, obtained by rescaling the energy axis $E$ of the calculated spectra as $E^{\prime}=E(1+\alpha)$, where $\alpha$ is approximately 0.05 for the $L_{3}$ edge and 0.07 for the $L_{1}$ edge. This rescaling brings the calculated features in agreement with the measured ones, that is, the error $\Delta E=\alpha E$ in the placement of the high-energy features is found to increase linearly with energy. On the other hand, according to calculations of Hedin and Lundqvist the shift due to self-energy corrections should decrease as $E^{-1 / 2}$ with increasing energy. ${ }^{15}$ Thus we attribute the difference between the measured and computed high-energy features to the omission of the electron-core-hole interaction in the final state.

The single-particle calculations have been carried out for $\mathrm{Gd}, \mathrm{Tm}$, and $\mathrm{Yb}$. The results for $\mathrm{Tm}$ and $\mathrm{Yb}$ are quite similar to that of $\mathrm{Gd}$ and, therefore, have been omitted. In the calculation of the band states we have neglected the spinorbit coupling. This approximation gives $L_{3}$ and $L_{2}$ spectra which have the same shape. The resulting single-particle ratio of the $L_{3}$ to the $L_{2}$ absorption is 2.14 for $\mathrm{Gd}, 2.16$ for $\mathrm{Tm}$, and 2.17 for $\mathrm{Yb}$. The departure of this ratio from 2 is entirely,due to the difference between the matrix elements involving the $2 p_{3 / 2}$ and the $2 p_{1 / 2}$ core states. The experiments which, within $10 \%$, are consistent with these values permit the neglect of spin-orbit coupling.

We emphasize that the width and height of the white line in all three cases are well described by the ground-state potential. This is also true for the high-energy cross sections which agree with the experimental ones within the uncertainty of the foil thickness of about $10 \%$. At the white line, the excited electron is promoted into a rather localized $5 d$ state, so that the core hole remains well screened and probably no major redistribution of the valence electrons occurs.

Electric-dipole selection rules allow transitions from the $2 p$ core levels into conduction states having both $s$ and $d$ partial-wave character. However, for the purpose of the following discussion the $s$ partial-wave contribution in the $L_{3}$ spectra can be ignored because of the much smaller $2 p$ $\rightarrow \epsilon S$ matrix elements compared with the $2 p \rightarrow \epsilon d$. Thus, the observable structure of the $L_{1}$ and $L_{3}$ spectra arise from strong features in the $p$ and $d$ projected densities of states. ${ }^{16}$ These projected densities mix strongly at high energies: Observe that the peaks in the $L_{3}$ spectra are associated with valleys in the $L_{1}$ spectra. In an extended xray absorption fine-structure (EXAFS) approach this corresponds to a phase shift of $\pi$ between the $s$ to $p\left(L_{1}\right)$ and $p$ to $d\left(L_{2,3}\right)$ terms. ${ }^{17}$ While one might distinguish between the high-energy EXAFS oscillations and the white line which appears as a matrix element effect (due to a different wavefunction normalization), both approaches yield the same final result.

The dominant single-particle feature on the low-energy side of the spectra is the large $5 d$ density of states, which gives rise to the white line. We point out, however, that in that energy region the $2 p \rightarrow \epsilon d$ matrix elements are strongly energy dependent and that there is no simple connection between the number of unoccupied $5 d$ states and the area under the white line. In Fig. 2 , we have also indicated the position of the $5 f$ band, which lies some $20 \mathrm{eV}$ above the Fermi level due to the large centrifugal contribution $l(l+1) / r^{2}$ to the potential for $f$ waves.

We gratefully acknowledge the valuable assistance of V. O. Kostroun in the early stages of this project. This work was supported in part by the Materials Science Center, Cornell University, under a grant from the National Science Foundation.

\footnotetext{
(a) Present address.

${ }^{1}$ R. D. Leapman and L. A. Grunes, Phys. Rev. Lett. 45, 397 (1980).

${ }^{2}$ H. Launois, M. Rawiso, E. Holland-Moritz, R. Pott, and D. Wohlleben, Phys. Rev. Lett. 44, 1271 (1980).
} 
${ }^{3}$ R. M. Martin, J. B. Boyce, J. W. Allen, and F. Holtzberg, Phys. Rev. Lett. 44, 1275 (1980).

${ }^{4}$ A. Bianconi, M. Campagna, S. Stizza, and I. Davoli, Phys. Rev. B 24, 6139 (1981).

${ }^{5}$ G. Krill, J. P. Kappler, A. Mayer, L. Abadli, and M. F. Ravet, J. Phys. F 11, 1713 (1981).

${ }^{6}$ See, for example, H. B. Stuhrmann and H. Notbohm, Proc. Natl. Acad. Sci. 78, 6216 (1981).

${ }^{7}$ V. L. Moruzzi, A. R. Williams, and J. F. Janak, Calculated Electronic Properties of Metals (Pergamon, New York, 1978).

${ }^{8}$ G. Materlik and V. O. Kostroun, Rev. Sci. Instrum. 51, $86(1980)$.

${ }^{9}$ J. E. Müller, thesis, Cornell University, 1980 (unpublished); J. E. Müller and J. W. Wilkins, to be published. This method is specially designed to solve the energy-band problem over an extended energy range and can describe simultaneously several principal quantum numbers of the eigenstates. Detailed absorption spectra calculations for the $3 d$ and $4 d$ transition metals have already been published: J. E. Müller, O. Jepsen, and J. W. Wilkins, Solid State Commun. 42, 365 (1982); J. E. Müller, O. Jepsen, O. K. Andersen, and J. W. Wilkins, Phys. Rev. Lett. 40,720 (1978).

${ }^{10}$ The potential was calculated by the standard prescription of L. F. Mattheiss, Phys. Rev. 133, A1399
(1964). Self-consistency, checked for the case of Gd, only makes minor changes in the placement of the $5 d$ band.

${ }^{11}$ O. Keski-Rahkonen and M. O. Krause, At. Data Nucl. Data Tables 14, 139 (1974).

${ }^{12}$ I. Lindau and W. E. Spicer, J. Electron Spectrosc. Relat. Phenom. $\underline{3}, 409$ (1974).

${ }^{13} \mathrm{~A}$ similar study performed for the $K$-edge spectra of the $3 d$ metals showed no appreciable discrepancies between theory and experiments: J. E. Müller, B. Lengeler, G. Materlik, and J. W. Wilkins, to be published.

${ }^{14}$ U. von Barth and S. Grossmann, Phys. Scr. 21, 580 1980 ; G. D. Mahan, Phys. Rev. B 21, 1421 (1980). The data presented here might be viewed as evidence for the "final-state rule" which claims that one-particle calculations can give realistic $\mathrm{x}$-ray absorption spectra provided final-state energies and wave functions are calculated in the presence of the core-hole potential.

${ }^{15}$ L. Hedin and B. I. Lundqvist, J. Phys. C $\underline{4}, 2064$ (1971) For an early application to Ni metal, see R. E. Watson, J. F. Herbst, L. Hodges, B. I. Lundqvist, and J. W. Wilkins, Phys. Rev. B 13, 1463 (1976).

${ }^{16}$ Müiller, Jepsen, Andersen, and Wilkins, Ref. 9.

${ }^{17}$ See, for instance, P. A. Lee, P. H. Citrin, P. Eisenberger, and B. M. Kincaid, Rev. Mod. Phys. $\underline{53}, 769$ (1981).

\title{
Superconductivity in a New Family of Organic Conductors
}

\author{
S. S. P. Parkin, E。 M. Engler, R. R. Schumaker, R. Lagier, V. Y. Lee, \\ J. C. Scott, and R. L. Greene \\ IBM Research Laboratory, San Jose, California 95193
}

(Received 8 November 1982)

\begin{abstract}
Evidence for superconductivity in (BEDT-TTF) ${ }_{4}\left(\mathrm{ReO}_{4}\right)_{2}$ [ where BEDT-TTF is bis (ethylenedithiolo) tetrathiafulvalene] near $2 \mathrm{~K}$ for pressures above $4 \mathrm{kbar}$ is reported. This is the first unambiguous observation of superconductivity in a sulfur-donor organic system. At higher pressures the transition temperature decreases rapidly, $d T_{c} / d P \sim-0.3$ $\mathrm{K} /$ kbar. At low pressures this material exhibits a first-order metal-insulator transition, postulated to result from a change in the arrangement of the anions which are ordered at room temperature.
\end{abstract}

PACS numbers: $74.10 .+\mathrm{v}, 61.65 .+\mathrm{d}, 71.30 .+\mathrm{h}, 72.15 . \mathrm{Eb}$

We report the discovery of superconductivity in a new family of organic conductors based on a sulfur-donor organic molecule. This is only the second organic system in which superconductivity has been observed. The overwhelming majority of organic conductors are unstable at low temperatures to periodic lattice distortions leading to an insulating ground state. ${ }^{1}$ Over the past decade extensive studies on a wide range of structural and chemical types of organic linear-chain systems were carried out with the important aim of suppressing these instabilities and stabilizing a low-temperature metallic or superconducting state. Recently this goal was finally achieved for several members of the (TMTSeF $)_{2} X$ family of compounds (where TMTSeF is tetramethyltetraselenafulvalene and $X$ is, for example, $\mathrm{PF}_{6}$, $\mathrm{TaF}_{6}, \mathrm{ReO}_{4}, \mathrm{ClO}_{4}$ ) with the observation of superconductivity near $\sim 1 \mathrm{~K}$ for pressures above some critical pressure, $P_{c} \sim 1$ bar to 12 kbar. $^{2-5}$ The reason why these particular compounds become superconducting is unclear, although it may be related to their atypical crystal and chemical structure. Indeed the related isostructural series 